\title{
Hypertension at high altitude: the interplay between genetic and biochemical factors in the setting of oxidative stress
}

\author{
Mohammad Z Ashraf \\ Hypertension Research (2016) 39, 199-200; doi:10.1038/hr.2015.140; published online 10 December 2015
}

$\mathrm{E}$ ssential hypertension, which is a complex

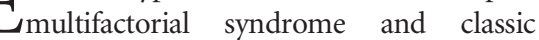
cardiovascular disease risk factor, contributes to adult morbidity and mortality worldwide each year. The physiological regulation of blood pressure (BP) is primarily dependent on the complex interactions between environmental and genetic factors. The past three decades have witnessed remarkable progress in the study of hypertension as a result of single-locus research and genome-wide association studies; however, the common predisposing genetic markers of the disease have yet to be established. The failure to identify the markers of hypertension may be the result of the smaller sample sizes used for the aforementioned association studies, the lack of replicated studies involving cohorts of either the same or different ethnicities, the lack of gene-gene interaction studies or the inappropriate selection of markers for the aforementioned association and interaction studies.

Oxidative stress (OS) is a multisystem phenomenon that is involved in the regulation of $\mathrm{BP}$, and affects the heart, kidneys, nervous system, vasculature and immune system. Moreover, OS accompanied by decreased nitric oxide levels in the brain results in sympathoexcitation, which has an important role in the pathogenesis of hypertension. ${ }^{1}$ The findings published by Kumar et al. ${ }^{2}$ elegantly demonstrate the relationship between CYBA (p22phox) genetic variants and their haplotypes in the setting of essential hypertension in both

MZ Ashraf is at Division of Genomics, Defence Institute of Physiology \& Allied Sciences, Lucknow Road, Delhi, India.

E-mail: mohammadzashraf@gmail.com high- and low-altitude populations. The selection of two populations in this study seems appropriate because hypertension results in more than seven million deaths every year; the incidence among lowlanders worldwide is increasing. ${ }^{3,4}$ However, the epidemiological data regarding the prevalence of hypertension in lowlanders who reside temporarily at high altitudes or native highlanders who live under stressed conditions are scarce. Furthermore, given these extreme environmental conditions, the selection of candidate genes related to OS, such as CYBA (p22phox), also seems appropriate; however, the relationship between CYBA (p22phox) and hypertension has been questioned in previous studies.

There is evidence to suggest that OS has a central role in the progression of both clinical and experimental forms of hypertension., OS results from increased production of reactive oxygen species and decreased levels of antioxidants, such as superoxide dismutase, catalase (CAT) and vitamins A, $\mathrm{C}$ and $\mathrm{E}$, in the setting of hypertension. Recent studies have also suggested that altered renal CAT and glutathione peroxide mRNA expression and activity precede the development of hypertension in spontaneously hypertensive rats. ${ }^{7}$ Kumar et al. demonstrated that the downregulation of CAT and the upregulation of 8-iso-prostaglandin F2 $\alpha$ (8-isoPGF2 $\alpha$ ) may represent promising markers of oxidative injury among hypertensive individuals. Diminished CAT activity results in excessive reactive oxygen species production and elevated 8 -isoPGF $2 \alpha$ levels in these patients. The oxidant-antioxidant imbalance in hypertensive patients documented by Kumar et al. demonstrated a shift toward increased oxidant levels, a finding that is suggestive of its possible contribution to the pathogenesis of the disease. Of note, aberrant OS-mediated redox signaling causes inflammation, hypertrophy, apoptosis, migration, fibrosis, angiogenesis, endothelial dysfunction and severe vascular remodeling in the setting of hypertension. ${ }^{8}$ Furthermore, the inter-individual susceptibility to hypertension and varied responses to targeted therapies may be explained by both genotype and phenotype correlations. Interestingly, Kumar et al. observed that an increased risk of hypertension exists among patients carrying the maximum number of risk alleles compared with patients carrying fewer numbers of risk alleles (Figure 1). However, the patients with protected alleles exhibited a reduced susceptibility to hypertension. The risk or protection conferred by individual alleles or haplotypes has a prominent role in both the functional and the molecular mechanisms underlying pathophysiology of the disease. ${ }^{9,10}$ Furthermore, the hypertension associated risk haplotypes correlated with decreased CAT activity, increased 8 -isoPGF2 $\alpha$ levels, increased systolic BP, increased diastolic BP and increased mean arterial pressure. Conversely, the protective haplotypes were associated with increased CAT activity, decreased 8 -isoPGF2 $\alpha$ levels and decreased clinical parameters, highlighting the functional relevance of $C Y B A$ (p22phox) gene variants in hypertension.

The possible involvement of OS in the underlying pathology of the disease is not without controversy. There exists a large amount of evidence suggesting that OS may not be the causal mechanism of hypertension. In fact, it has been suggested that 


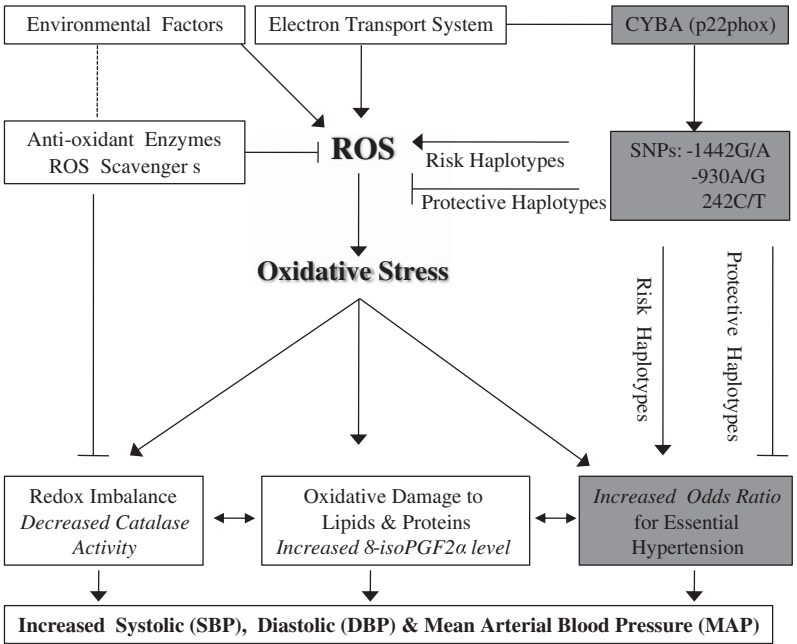

Figure 1 Diagrammatic representation for association of CYBA (p22phox) polymorphism with pathophysiology of essential hypertension. A full colour version of this figure is available online at the Journal of Hypertension Research website.

hypertension may cause OS. ${ }^{11}$ Therefore, the possibility that $\mathrm{OS}$ is involved in the development of additional altitude related maladies is considerable. Conventional wisdom assumes the efficacy of 'antioxidant therapies' in counteracting high-altitude maladies. However, Julian et al. ${ }^{12}$ recently questioned this hypothesis. They determined that hypobaric hypoxia-enhanced enzymatic antioxidant systems in subjects prone to acute mountain sickness. They proposed that quenching oxidant activity may exert adverse downstream effects. Whether such a phenomenon exists in the case of hypertension remains unknown.

In conclusion, the study by Kumar et al. may have added a new dimension to the study of hypertension, particularly at high altitudes; however, studies with larger sample are necessary to develop specific biomarkers and effective disease management strategies.

\section{CONFLICT OF INTEREST}

The author declares no conflict of interest.

1 Kishi T. Regulation of the sympathetic nervous system by nitric oxide and oxidative stress in the rostral ventrolateral medulla: 2012 Academic Conference Award from the Japanese Society of Hypertension. Hypertens Res 2013; 36: 845-851.

2 Kumar R, Kohli S, Ali Z, Duhan K, Ram R, Gupta M, Tyagi S, Mohammad G, Pasha MAQ. CYBA (p22 ${ }^{\text {phox }}$ ) variants associate with blood pressure and oxidative stress markers in hypertension: a replication sizes containing additional genetic variants study in populations of diverse altitudes. Hypertens Res 2015; 38: 498-506.

3 Kearney PM, Whelton M, Reynolds $K$, Muntner $P$, Whelton PK, He J. Global burden of hypertension: analysis of worldwide data. Lancet 2005; 365: 217-223.

4 Danaei G, Finucane MM, Lin JK, Singh GM, Paciorek CJ, Cowan MJ, Farzadfar F, Stevens GA, Lim SS, Riley LM, Ezzati M. Global Burden of Metabolic Risk Factors of Chronic Diseases Collaborating Group (Blood Pressure). National, regional, and global trends in systolic blood pressure since 1980: systematic analysis of health examination surveys and epidemiological studies with 786 country-years and 5.4 million participants. Lancet 2011; 377: 568-577.

5 Touyz RM, Briones AM. Reactive oxygen species and vascular biology: implications in human hypertension. Hypertens Res 2011; 34: 5-14.

6 Tomaselli GF, Barth AS. Sudden Cardio Arrest: Oxidative stress irritates the heart. Nat Med 2010; 16: 648-649.

7 Sundaram A, Keah LS, Sirajudeen KNS, Singh HJ. Upregulation of catalase and downregulation of glutathione peroxidase activity in the kidney precede the development of hypertension in pre-hypertensive SHR. Hypertens Res 2013; 36: 213-218.

8 Schriner SE, Linford NJ, Martin GM, Treuting P, Ogburn CE, Emond $\mathrm{M}$, Coskun PE, Ladiges W, Wolf N, Van Remmen H, Wallace DC, Rabinovitch PS. Extension of murine life span by overexpression of catalase targeted to mitochondria. Science 2005; 308 : 1909-1911.

9 Wyche KE, Wang SS, Griendling KK, Dikalov SI, Austin $\mathrm{H}$, Rao S, Fink B, Harrison DG, Zafari AM. C242T CYBA polymorphism of the NADPH oxidase is associated with reduced respiratory burst in human neutrophils. Hypertension 2004; 43: 1246-1251.

10 Kumar R, Kohli S, Mishra A, Garg R, Alam P, Stobdan T, Nejatizadeh A, Gupta M, Tyagi S, Pasha MA. Interactions between the genes of vasodilatation pathways influence blood pressure and nitric oxide level in hypertension. Am J Hypertens 2015; 28: 239-247.

11 Montezano AC, Touyz RM. Oxidative stress, Noxs, and hypertension: experimental evidence and clinical controversies. Ann Med 2012; 44: S2-16.

12 Julian CG, Subudhi AW, Hill RC, Wilson MJ, Dimmen AC, Hansen KC, Roach RC. Exploratory proteomic analysis of hypobaric hypoxia and acute mountain sickness in humans. J Appl Physiol 2014; 116: 937-944. 